\title{
XXXVI. On the repulsive power of heat
}

\section{H.F. Talbot Esq. F.R.S.}

To cite this article: H.F. Talbot Esq. F.R.S. (1836) XXXVI. On the repulsive power of heat, Philosophical Magazine Series 3, 8:46, 189-191, DOI: 10.1080/14786443608648845

To link to this article: http://dx.doi.org/10.1080/14786443608648845

$$
\text { 曲 Published online: } 01 \text { Jun } 2009 .
$$

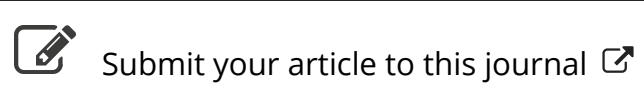

\footnotetext{
Џll Article views: 2
} 
also be devoted to the publication of the monthly results (according to a fixed tabular form) of observations taken in various parts, not only of this kingdom, but of the whole world, if possible. I am, Gentlemen, yours obediently, Joseph Atrkinson,

Carlisle, Dec. 1, 1835. Sec. of the Carlisle Lit. and Phil. Soc.

XXXVI. On the repulsive Power of Heat. By H. F. TALBoT, Esq., F.R.S.*

Experiment 1.-On the Vaporization of Sulphur.

WHEN a minute portion of sulphur is warmed between two plates of glass, it sublimes, and forms gray nebulous patches, which are very curious microscopic objects. Each cluster consists of thousands of transparent globules, exactly imitating in miniature the nebulæ which we see figured in treatises on astronomyt. By observing those particles which are larger than the others, we find their figure not to be spherical, but plano-convex, with the flat side to the glass. Being very transparent, each of them acts the part of a little lens, and forms in its focus the image of a distant light, which can be perceived even in the smaller globules until it vanishes from minuteness. If they are examined again after a certain number of hours, the smaller globules are generally found to retain their transparency, while the larger ones are become opake, in consequence of some internal change in the arrangement of their molecules. I find that Mitscherlich and others have noticed this property in sulphur of undergoing a spontaneous changet. There is a circumstance attending this experiment which deserves particular attention. Although the sulphur has been sublimed by heating it over a lamp between two plates of glass almost in contact with one another, yet the globules are found adhering to the upper glass only; and as their number amounts generally to many thousands, it is evident that the preference which they thus exhibit to the upper glass must have some strong determining cause.

The reason of it is, no doubt, that the upper glass is a little cooler than the lower one; and by this means we see that the vapour of sulphur is very powerfully repelled $\S$ by heated

* Communicated by the Author.

+ And owing, perhaps, their mutual disposition to the same general laws of attraction as the Nebulæ?-EnIT.

$\ddagger$ See Phil. Mag. and Annals, N.S., vol, iii. p. 144, 152, for correlative facts.-EDit.

\$ This is a very beautiful instance in corroboration and extension of Prof. Powell's experiment described in the Phil. Trans. 1834, part ii. p. 485.-Enit. 
glass. The plano-convex form of the particles is owing to the force with which they endeavour to recede from the lower glass, and their consequent pressure against the surface of the upper one. I think this experiment is a satisfactory argument in favour of the repulsive power of heat, and I believe it has not been hitherto described.

\section{Experiment 2.-On the Vaporization of Arsenic.}

When a particle of arsenic is sublimed between two plates of glass, it forms nebulous patches, considerably resembling those formed by sulphur in the preceding experiment. But the microscope detects a great difference. Instead of a globular or semiglobular form, the particles of arsenic are crystallized. The minuteness of some of the crystals almost exceeds calculation. I would suggest the employment of this method to detect the presence of arsenic in minute quantities of matter. The difficulty of demonstrating its presence with sufficient certainty is shown by the number of chemical essays that have been written on the subject, while a particle of the size of a pin's head is amply sufficient to display this microscropic crystallization; and the form of the crystals being distinct and definite, the observer can soon make himseif acquainted with their figure, so as to run little risk of mistaking any other substance for them.

\section{Note on Radiant Heat.}

M. Melloni says (in the Number of this Journal for December last, vol. vii. p. 475, that

"For a long time the immediate transmission of terrestrial radiant heat by transparent substances, both solid and liquid, bas been denied; and the opinion has become prevalent that we see in experiments of this kind only an effect of the heat absorbed by the body submitted to the calorific radiation."

This "prevalent opinion" he bas shown to be erroneous, but by experiments which are too delicate to be repeated with facility.

As a popular illustration of the fact, therefore, seems to be wanted, I subjoin the following rude but convincing experiment.

Let a poker be heated bright red hot, and having thrown open a window, approach the poker quickly to the outside of a pane, and the hand to the inside. A strong heat is felt at the instant, which ceases as soon as the poker is withdrawn, and may be again renewed, and made to cease as quickly as before. Now, everybody knows that if a piece of glass is so much warmed as to convey this impression of heat to the 
hand, it will retain some part of that heat for a minute or more; but in this experiment the heat vanishes in a moment. It is not, therefore, heated glass which we feel, but heat which has come through the glass, in a free or radiant state.

XXXVII. Extracts from a Prize Essay on Iodine. By $\mathrm{J}_{\mathrm{AMES}}$ INGLIS, M.D.

[Continued from p. 20, and concluded.]

I MENTIONED before that Serullas had proposed iodic acid as a test for the vegetable alkalies. I find that hydriodic acid may be used as such also. To the sulphate of quinine $I$ added in solution a few drops of sulphuric acid, so that the sulphate might be soluble in water. I next added a solution of hydriodate of potassa ; instantly there was a yellow precipitation, which became gradually of a greenish colour. I added more hydriodate, and the precipitation of yellow iodide of quinine still took place, which finally became of a reddish brown colour. I have not examined this compound, but call it iodide of quinine, the hydriodates being all soluble; and I do not see how it could be an iodate.

I find also that the hydriodate of potassa throws down a white precipitate with tincture of capsicum. I cannot decidedly say that this acid is as good a general test as the iodic. * * * *

I wished to obtain a compound of cyanogen with iodine, and for this purpose made a solution of bicyanuret of mercury in water, which I added to an alcoholic solution of iodine; immediately the red biniodide of mercury fell, and the action I thought to be this:

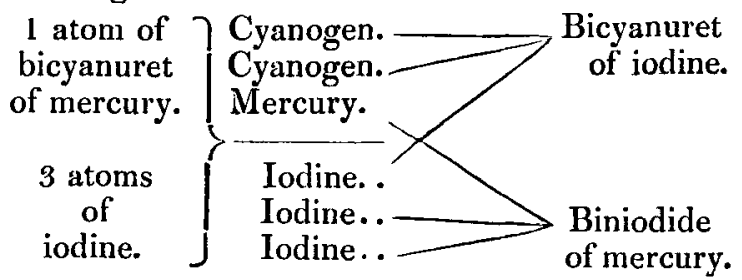

If too much cyanuret of mercury be added, then all the precipitation is red. But if there be only sufficient, then a ligbtish brown powder in crystals falls. I boiled and strained off the supernatant liquor from the biniodide of mercury, and laid it aside to crystallize; but this could not be effected. The liquid is exceedingly pungent, and exhales a vapour 